\title{
Exploratory Study of Smart Healthcare Services (e-Health \& m-Health Applications) in the UAE- Exploring Related Literature \& Websites Contents
}

\author{
Dr. Fathi Fayeq Salameh \\ Assistant Professor, Business Administration College, Al-Falah University \\ Dubai, United Arab Emirates
}

\begin{abstract}
This study highlights the importance and the growing trend of e-health in the modern world, with special emphasis on Dubai- UAE. The effectiveness and usability of mobile application tools and devices have also been described in this study. Moreover, the different components and features of a Health service for providing a service facility to the patient and to maintain and manage the health sector information and data have been discussed. However, the shortcomings and the weaknesses along with the disadvantage of e \& m-Health application are illustrated in this study. The purpose and usability of mobile application and the electronic devices for health tracking and monitoring are reported in this study. In addition, the future trend and the expectations of individuals and the patients related to the advancement in the e-health service are explained.
\end{abstract}

Keywords: UAE, e-Health, m-Health, Technology, Applications.

\section{Introduction}

In the era of the modern and busy world, many people don't usually have time for regular medical treatments and visits to doctors, which may imply longer waiting times either to arrange appointments or to wait in the reception area to finish registration procedures and to see their doctors. The information technology has changed the dimension and service facility of the Healthcare sector with management of information and data related to the patient and other Healthcare aspects for providing better assistance and service facilities. It allows the recording of details for patients by providing decision-related diagnosis and treatment (Venot et al, 2016). Moreover, the mobile application tools are rapidly growing in the market which is providing instant Health Care advice and support to the patients and the individual (Omer et al, 2015). The study mainly relied on theoretical descriptive approach based on exploring the secondary information related to the e \& m-health provided by other researchers and health \& governmental authorities in the UAE.

\section{What is E-Health?}

E-Health is considered to be a conjunction of medical Science with information technology for intersecting the medical informatics which provide health-related services with the use of the internet and other related Technology (Eysenbach, 2001). Eysenbach (ibid) also added that the term 'e-Health', in a broader sense, is characterized not only as a technical development, but also as a state-ofmind, a way of thinking, an attitude, and a commitment for networked, global thinking, in order to improve health care locally, regionally, and worldwide by using information and communication technology.

The definition of e-health by The 'World Health Organisation' (WHO) provides three basic techniques of addressing, The systems are used to disperse healthcare information to patients and clinicians via the Web or local IT, to make government health programs better and the technology is used by healthcare executives to improve the operation of the organisation (Al Ketbi and Al Deen, 2018).

E-Health is a recent practice which is performed by the Healthcare sector with the use and support of the electronic process and communication tools and devices for curing and prescribing medicine to the patient. However, it is also used as a recommendation tool in the form of a mobile application by collecting the details related to the symptoms of patients and then recommending medication to the patient.

\section{What is m-Health?}

Assisting patients, healthcare providers and policymakers are the key elements of the $\mathrm{m}$ Healthcare. Basically, it provides the patients with tools to observe their health changes and converts those alterations in the health state to the physician of the patients. It ensures the 
connectivity of the health providers with their colleagues and facilitates their communication (Zubair et al, 2016). In addition, m-health also aids the physicians to access various sources of information resource. The health delivery circumstances, and the medical outcomes are also being evaluated and estimated by the medical physicians by using the m-health tools. As per the definition, "any product, including accessories, used in healthcare for diagnosis, prevention, monitoring or treatment of illness or handicap excluding drugs. Medical devices can be consumables, diagnostic imaging, dental products, orthopaedice prosthetic products, and patient aids" (export.gov, 2018).

Furthermore, an early stage of growth has been observed in m-health in multiple countries. The tribulations in its development also prevail. The 'Hospitals, telecommunications operators, and health professionals' put in efforts to link mobile health devices and applications with the diagnosis and treatment. For expediting m-health development, necessary alterations and modifications in the policymaking practices shall be guaranteed (Harous et al, 2018).

\section{Essential Backgrounds}

Popularity has been observed in the growth of e-health, including m-health, services and tools. Analysts reported that the size of the worldwide mobile health market in the year of 2017 is 23 billion US dollars. They pointed out that Europe and Asia-Pacific will have the largest markets followed by North America (Jihad et al, 2016). In addition, the World Health Organisation (WHO) has undertaken a survey of 114 countries and concluded that various countries have adopted $m$-health. The establishment of the health call centres was the major activity in this regard; which facilitated the inquiries by the patients. The appointment reminders were being provided through Short Messaging Services (SMS) with the aim of facilitating the m-health services. Additionally, the provision of the telemedicine, access to patient records, measurement of the compliance of the treatment, raise in the health awareness, observing patients and in providing support to the physician to undertake decisions (Harous et al., 2018).

\section{Functions Types and Elements of E-Health}

'Electronic Health Application' should aid health professionals, patients and the public in different aspects. IMS Institute for Healthcare Informatics (2013, p. 9) identified seven major categories of health app functionalities:

\begin{tabular}{|l|l|}
\hline Inform & $\begin{array}{l}\text { Provide information in a variety } \\
\text { offormats (text, photo, video) }\end{array}$ \\
\hline Instruct & Provide instructions to the user \\
\hline Record & $\begin{array}{l}\text { Capture user entered data } \\
\text { Graphically display user entered } \\
\text { data/output user entered data }\end{array}$ \\
\hline Display & $\begin{array}{l}\text { Provide guidance based on user } \\
\text { entered information, and may } \\
\text { further offer a diagnosis, or } \\
\text { recommend a consultation with } \\
\text { a physician/a course of treatment }\end{array}$ \\
\hline Remind/Alert & Provide reminders to the user \\
\hline Communicate & $\begin{array}{l}\text { Provide communication with } \\
\text { healthcare } \\
\text { professionals (HCP)/patients } \\
\text { and/or provide links to social } \\
\text { networks }\end{array}$ \\
\hline
\end{tabular}

The types of e-Health and their elements are related to some categories. A few of them are listed below:

\section{Electronic Medical Records}

This assists Healthcare units in establishing an appropriate communication, related to the data of patients, amongst the professionals. When performing an electronic medical record (EMR), persistent information may include a period of time that is included in different health care procedures. It helps distinguish people who are expected to have tests or preventive tests, and to show how each patient meets their specific needs, such as: immunisations and pulse measurements. The goal of EMR is to enable provision of effective and accurate reviews. Perhaps the most surprising difference is that EMR recordings are common and patients in each clinic using EMR software have electronic sketches instead of having multiple charts in multiple clinics (Gallos and Mantas, 2017).

\section{m-Health}

m-Health applications have different sources. Aungst et al (2014) identified a number of these apps according to their sources as follows: 
- Native mobile apps: Software that comes preinstalled on a mobile device (e.g. software that operates a device's built-in camera).

- Downloadable mobile apps: Software that is not preinstalled on a device and must be downloaded from an external source, usually a mobile app store.

- Web-based app: Software that connects to an internet portal and displays content on a mobile device. Requires an internet connection.

Around two-thirds of the world population have a mobile connection and in order to please the emerging mobile health industry, individuals are engaged in maintaining their health issues by using their mobile phones for better gauging the symptoms in an everinnovative way (Bodolica and Spraggon, 2019). Harous et al (2018) mentioned that "almost 100,000 health-related apps have been added by 13,000 new publishers to the market since 2015. At that moment, there had already been 259,000 medical apps in major app stores". Zubair et al (2016) stated that regarding the term 'healthcare application', people generally assume that it would be a health/fitness tracker, but these applications are much more than only being a fitness tracker. Smartphones are an easily accessible and this has contributed not only for the mobile sector but also a major driver of the healthcare industry (Zubair et al, 2016).

There are few applications which have great importance in daily life for keeping health \& fitness maintained. 'Samsung health' is one of them which is a free mobile application available for Smartphone users. The application is helpful in tracking several aspects of daily activities such as sleeping, dieting, self-control \& physical activity etc. The application is greatly effective in tracking diet control and has an auto-detection of the workout by recognising the movements during walking or running (Samsung, 2019). 'Pill reminder' is another application tool which is highly effective in reminding the patient to take their medicine and pills at the right times (Mack, 2017). However, the application is also effective for keeping track of glucose levels, blood pressure, and other health-related measurements. 'Water drinking reminder' is an effective application tool for reminding us to take water at the recommended time by giving instructions about the amount of water that needs to be taken at regular intervals. (Bhatt and Peddoju, 2016).

Mobile devices allow health professionals and patients to carry out health and medical practices and procedures. Patients can monitor and extract information with the use of mobile devices or other wireless devices (Paho eHealth, 2019). m-Health also helps patients talking to their doctors and care groups to meet directly through the application. Safe information, as an element of $\mathrm{m}$-Health, also enables physicians and healthcare providers to talk to each other about patients' affairs (Samihah, 2019).

\section{Health Informatics}

It is a software-based supporting tool which provides a solution for managing the data of the patient, making an appointment, administrating tasks and management of work schedule. This tool includes computer science, choice of support networks, telemedicine, morality, global health computing, translational research and home care. Scientists will find ways to plan and execute imaginative applications and develop new technologies to improve medical care (Henderson, 2019).

\section{E-prescribing}

This allows the Healthcare unit to make prescription in an electronic format and transfer it to the pharmacist or to the patient via electronic means with the use of the internet. In the simplest case, the structure of the electronic recipe is entered as an electronic reference manual. The progressive structure of the electronic prescription acts as the author of individual resources. You can create and supplement therapies for individual patients, monitor prescriptions and display a tolerant history, interact with the pharmacy or other drug-delivery sites, and incorporate electronic patient record (EPR) structures (Mack, 2017).

\section{Benefits of Technologies}

The benefits that these technologies can bring are varied since, for decades, a person's relationship with their own health was primarily a list of doctors, institutions, hospitals and payers firmly in charge of making a decision (Gallos \& Mantas, 2017). Today, however, the individual has 
much more control of the decisions and changes they can bring to their habits. Appropriate use of technology in health can improve outcomes through prevention, favouring quality and life expectancy (Benharref \& Serhani, 2013).

In addition to be an irreversible trend, applications for general health and well-being help in coordinating the care of the individual. Alloghani, et al (2015) pointed out that these technologies allow the patient, for example, to have greater power in manging their dietary needs to combat overweight and high cholesterol. Mack identified another benefit, which is the integration of patient data, making information such as immunisation records, exam results and medical history available and accessible (Mack, 2017).

Sharing experiences amongst app users of this nature through social networks is potentially useful for improving adherence to treatments and disseminating qualified information about certain health conditions, beyond the borders of a hospital or clinic (Henderson, 2017).

Through smart apps it became possible to solve simple and timely patient doubts, which with the vast majority of them can be answered via email or telephone, thus helping the doctor-patient approach (Venot et al, 2016). Such technologies must be used in parallel with real and face-to-face healthcare. Alloghani, et al (2015) pointed out that it is important to note that these applications do not replace consultation and guidance from a professional, such as a doctor or nutritionist. In addition, it is necessary to check with caution the source and reliability of the information offered by this app.

With the increasing progress of these technologies, those who are able to combine health and connectivity, see the concern that people are having. More focus on improving their living habits, only tends to win, including companies that have already seen this advance in favour of their patients and clients, seeking health care, information and holistic well-being. Any entity that chooses to ignore these technological developments runs the risk of becoming obsolete.

But despite this, data from an analysis conducted by Kantar in the United States on the health technology situation, indicates that only $30 \%$ of American physicians have already recommended health and wellness or wearables apps to their patients (Alloghani, et al., 2015).

On the user side, the perception is different. Almost half of Americans surveyed understand the positive use of technology for health. The study integrated information from several surveys conducted by the Institute last year (Samihah, 2019). In the UK, $29 \%$ of people use mobile applications and wearable devices to monitor their own health (Al Ketbi\& $\mathrm{Al}$ Deen, 2018). The data is from an international survey conducted by GfK and conducted with more than 20 thousand internet users from 16 countries (Benharref \& Serhani, 2013). Around 4,900 people were interviewed. According to the numbers, the country is already side by side with the United States when it comes to the use of these resources (Paho e-Health, 2019). The numbers tend to increase. According to Gartner, the wearables market is expected to grow $16.7 \%$ per year, reaching $\$ 34$ billion by 2020 . Estimates point to more than 830 million connected devices in 2020 (Gallos \& Mantas, 2017).

\section{Health Indicators and Technological Advances}

An alarming state of growth in chronic diseases has been observed in the world, specifically in the UAE. The UAE ministry of health published statistics which proposed that $25 \%$ of deaths were being observed in the country due to the chronic diseases (Benharref and Serhani, 2013); In particular, the cardiovascular diseases. In addition, huge divergent factors, including "cholesterol, diabetes, high blood pressure, physical inactivity, smoking, overweight and obesity"; but not only these specifically (Harous et al., 2018). To cater this increase in the morbidities by these chronic diseases and the related impediments, specifically formulated and well-organised monitoring and "monitoring and prevention approaches" could aid in the risk-taking of the occurrence of these diseases by eliminating the key causes of the cardiovascular diseases (Zubair et al., 2016). The advances in the healthcare systems have triggered the convergence of the prior mortal diseases into the chronic diseases now. This has assisted in fulfilling the demand of continuous care especially in the cases of elderly people. "In terms of medical devices, 
the U.A.E. is an import driven market that is growing rapidly to keep pace with the country's expanding healthcare infrastructure. The government and private healthcare sector are investing heavily to provide countrywide healthcare solutions to the residents, expats, and medical tourists. Orthopaedic devices are expected to register the highest growth specifically due to the aging population" (Export.gov, 2018).

However, the over-growing expenses for both the patients and the caretakers are leading to a certain degree which may cause the scarcity of the e-healthcare services. In this regard, the evolving tradition of the e-healthcare services assists the patients and the healthcare providers' inconvenient access to significant health information anywhere and anytime (Paho e-Health, 2019).

e-Health applications have been growing at the same rate as technological advancement: since 2015, the use of these apps has increased by $25 \%$, according to a recent survey by Kantar (Mack, 2017). And not only through smartphones, but also through dressing technologies: so-called wearable gadgets, which grew by $12.3 \%$ in American consumption - today $21 \%$ of adults in the United States report having a mHealth for the purpose of monitoring their health (Henderson, 2017). There are many different modalities on the market, ranging from devices that help control glucose, blood pressure and "Body Mass Index" (BMI), to those that accompany racing performance, calorie consumption, daily water quantity, feeding, menstrual period, amongst many others (Paho e-Health, 2019).

The study conducted by Kantar during 2017 and 2018 showed that nearly half of the consumers interviewed stated that they have a positive opinion on the technologies used for health (Samihah, 2019). However, almost 60\% are still not familiar with some of the more common devices (Venot, et al., 2016). "The healthcare area is clearly evolving towards a greater consumer focus and these devices will be at the forefront and centre of this movement," says Lynnette Cooke, Global CEO (Henderson, 2017).

Emergence of Electronic Health (e-Health) in UAE
Both the federal and Emirate levels in the UAE has regulated healthcare. "Federal-level legislation dates back to the 1970s and 1980s and there are pending legislative reform initiatives to facilitate the development of the healthcare industry. The U.A.E. Government is liberalising policies to attract foreign investments in order to improve the healthcare standard and boost the healthcare industry" (export.gov, 2018).

In the last few decades, UAE is moving rapidly from traditional medicine to robot medicine. Employing the most modern and advanced tools, instruments and applications is well known in different health organization and authorities. The Ministry of Health \& Prevention has undertaken a wide-reaching program of digital transformation, which aims to empower healthcare professionals, providers and patients, and build the foundation for a healthier nation

According to the news report article of 'Khaleej Time' and other sources, UAE is implementing digital technology in the Healthcare sector for making the Health Care unit more advanced in the digital world. eHealth is gaining importance in UAE and the nation wants to act as a catalyst in the context of integrating digital technology in the health sector. UAE has launched several mobile applications and wearable Healthcare devices for providing a better solution for the healthrelated challenges (Clarke, 2015). UAE is emphasising more implementing technology in the Healthcare sector to enhance its facility of healthcare services by using electronic means and digital media (Harous et al., 2018).

Access to medical services is a requirement for everyone through every stage of life. Improvements to healthcare systems can bring the most meaningful benefits to people everywhere. Therefore, healthcare is one of the key pillars for government digitalisation projects, both in how governments manage their healthcare, and how those medical services are delivered.

In the UAE, the Ministry of Health \& Prevention (MOHAP) is responsible for all areas of public healthcare and policy, and coordination with the private sector. The digital transformation journey for MOHAP covers many different aspects of the healthcare system, with integration and interaction across 
many of the different stakeholders in the UAE sector.

\section{Mobile Health (m-Health) Applications in UAE}

Smartphones and tablets can now be used to access medical records and check on hospital appointments under new measures to improve patients' access to health information. The modernisation of online services by Dubai Health Authority (DHA) is the latest move towards a paperless environment, a key focus of the Smart Government initiative. Furthermore, according to the vision, most of the government and private institutes in the UAE are concentrated in e-Health as their development plan of healthcare sector in the country. Many plans have been set and actions have been taken in this regard (mohap, 2019):

- Dubai Health Authority holds digital transformational forum 2016, which was about healthcare IT transformation, opportunities and challenges.

- Electronic Patient Medical Record such as SALAMA system in 2017, which means an accessible patient electronic medical file through all DHA facilities.

- UAE E Health week on March 2019, more than 300 health IT stakeholders discussions, stories, and international e health innovations.

- Etisalat Digital Healthcare Solution includes Smart Hospital, Health Information Framework, and population health Management.

Two new apps, Dubai Doctors and Sehhaty, will offer 93 smart services linked to the DHA website (DHA, 2019).

The modernisation will also help medical professionals, making it easier to issue and renew licences for doctors and facilities. The new e-services can be downloaded now from online app stores and are compatible on all mobile devices. Patients will be able to cancel or reschedule an appointment, renew health cards or request a duplicate, view lab results, medication information and apply for a medical report.

'MyMedicNow' is the healthcare application that has been recently introduced in UAE to assist in associating patients and physicians. Assistance in looking for the medical conditions along with helping patients in finding "local healthcare providers".
According to the founders of 'MyMedicNow': "The launch is timely as UAE's mobile health industry is positioned for a strong growth" (Tradearabia.com, 2019). m-health application allows the patient to connect with the precise physicians by assisting them to search the symptoms of the disease or disorder through an exclusive methodology. This newly introduced application helps the patient in searching for adequate doctors by searching for the medical conditions and to locate the nearby or local healthcare practitioner efficiently. IOS, Android and web portal are the only portals facilitating the m-health application currently (Gallos and Mantas, 2017).

Dhaval Desai, CEO of MyMedicNow stated: "From life-saving medications to cutting-edge treatments, the healthcare industry thrives on constant innovation" (Henderson, 2017). Henderson stressed that the biggest recent breakthrough in healthcare sector is the smartphone and its applications. He continued by saying, "there's an app for everything, but the best ones are the apps that'll get you out of a bind when emergency strikes (ibid). MyMedicNow is abounded with convenient solutions that not only help improve people's lives but also helps make informed choices easier and faster whilst ensuring doctors and hospitals are better connected to patients. This will reduce burden on hospitals (Samihah, 2019).

\section{Accessibility of the Applications}

In the era of modern technology and the smart world, these applications are easily accessible for smartphone users on application stores. In the context of usability, the applications are programmed and designed with a userfriendly approach so that it can be convenient for every person to use the application ( $A l$ Ketbi and $A l$ Deen, 2018). However, the application comes with an electronic user guide manual which is also effective in providing guidance to the new user in terms of operating the application. The co-founder of the Pulse Middle East reported that "The UAE has incredibly high smartphone penetration, which affects how residents in the UAE consume digital media and applications" (Khaleej Times, 2019). In addition, they reported that this application has been downloaded by 40,000 individuals with a panel of 4000 doctors on-board within the first quarter after its launch. The number of 
downloads was being marked as a great turnover of people by the founders of the MyMedicNow application (Henderson, 2017).

It usually depends on the scope of the application and its almost one out of four applications are capable of engaging the users. Even the doctors have registered themselves on the application as it has facilitated the need of the middle man and now the doctor and the physician can connect to each other directly. Thus, communication between the doctor and patient becomes convenient (Paho e-Health, 2019).

\section{Purpose of such Applications and Devices}

In the modern civilised world, people become very busy with their daily schedules and activities and they do not get real time for a health check-up. In addition to that, there are several regions where accessing a medication facility in an emergency is very hard due to the distance barrier and/ or long waiting times. Patients' admissions in hospitals, places an extensive burden on the provision of the services by the healthcare sector and this can be conveniently manageable if the health of the patients is being tracked by the m-health applications (Samihah, 2019). The formulation and implementation of a high-class infrastructure to facilitate the healthcare sector has become the main concern of the Government of the United Arab Emirates (UAE). The consequences of these efforts have caused UAE to suffer due to a shortage of trained medical personnel. There are numerous hospital construction and renovation programs underway as a result of public and private investment. "A growing medical tourism sector is generating demand for modern facilities with state-of-the-art medical equipment. Medical device production will continue to be low, and only limited to basic items such as various types of syringes, and IV sets will be locally produced" (Export.gov, 2019). This sector has been an advanced and modernised sector during the past few years.

In the age of modern technology, the internet has played vital role in providing Healthcare assistance to individuals and patients. These applications and devices are designed and programmed for regular use to monitor and detect the basic health issues. This will allow patients and the individual to track their health-related issues on day-to-day base, so that they can take their medication and receive medical assistance at the recommended time without being failed. In addition to that, small issues can create unconsciousness amongst the individual and it will create a state of tension amongst the family members (Benharref \& Serhani, 2014). Mobile-based application tools and devices provide a convenient medium to identify the issues related to health and are effective for self-treatment. Moreover, the application also allows one to consult with the doctor via video conference or through chat in order to take their doctor's recommendation and advice (Venot, et al., 2016).

\section{Expectations regarding the Future Trend and Invention of Healthcare Sector}

Research shows that there is a tremendous opportunity for health technology waiting to be explored, and with it the potential to revolutionise patient care, said Jennifer Carrea, CEO of Lightspeed Americas (Gallos, 2017). What is needed is a more effective education of patients and physicians (Al Ketbi \& Al Deen, 2018).

Terms such as artificial intelligence, machine learning and predictive analytics are increasingly common in the health world nowadays. Health Techs' investments in the development of devices that enable better management of people's health, especially medical information of individuals, are also growing worldwide (Paho e-Health, 2019).

A lot of technology industries and the health care units are working collaboratively to bring more advancement in the sector of digital Healthcare. The current market is blooming with the use of digital Healthcare devices and mobile application tools for getting instant help and support related to health care issues. Apart from that, mobile application tools are not efficient and effective enough to fulfil the expectation of the users and the patients. The technology industries and the Healthcare sectors are giving more effort to leverage the service facility of the Healthcare sector with the use of digital technology (Mack, 2017).

For the future purpose, the blockchain technology and artificial intelligence systems are integrating into the Healthcare units to provide real-time response related to the health care issues with the use of mobile applications and tools. Furthermore, the technological solutions are integrating into the 
health application tools and devices to share the data and information on a real-time basis by increasing the flexibility (Moumtzoglou, 2014). Human beings are becoming more concerned about their health and are introducing advancements in the digital Healthcare. This service will provide a better opportunity to the health sector for enhancing the service facility whilst fulfilling the expectations of the patients and individual (Gallos and Mantas, 2017).

\section{Shortcomings and Weaknesses}

Faced with application flaws, all mobile-based tools do not provide an accurate answer for the patient. According to the application error, health-based application tools do not work with mobile and non-mobile customers. However, most application tools' language are English and it will be very difficult for the general population that do not speak English. Non-technical persons believe that their application is difficult to process due to their low complexity (Gallos and Mantas, 2017).

In terms of defects, the application tools provide no permanent and ongoing help. However, it is not expected that a suitable proposition will be determined by infections or serious disorders. The application is not effective in providing all the Healthcare related services and people often find it unworthy to use mobile applications for accessing the health care services facility (Alkraiji et al, 2014). The virtual interaction is not enough for providing a better solution to cure any disease or healthrelated issue. Additionally, despite the modernisation of the world and the availability of mobile healthcare applications; the need for evaluating the acceptance of the individual toward the mobile health application whilst considering their own healthcare issues remains a questionable concern (Harous et al., 2018).

People take their blood glucose test through the mile application but their information remains with them and does not reach the right place - the physician, who would analyse, track the changes occuring with the individual, and prescribe the medical treatment of medicine to them. Furthermore, varying applications collect their patients' information in various formats but they remain underutilised by not reaching the physician and then the patients have to bear the expenses of unnecessary duplicate tests (Mack, 2017).

However, we are now making efforts in designing and implementing the use of a mobile health application which can facilitate the screening process in order to conduct the diagnosis of the disease or disorder; by linking the patients with the doctors through their mobile phones (Samihah, 2019).

\section{Conclusion}

This study has shown that the UAE applied digitalisation (e-Health \& $\mathrm{m}$-Health) in different governmental aspects, including the health sector. The Health sector and m-health are receiving a lot of importance in the present world with efficient management of data and information related to the Healthcare services and are providing a better service facility. In addition, the mobile applications and tools are effective in terms of tracking and monitoring daily activities. Digital devices are pretty much effective in providing information on health-related issues.

The application and tools are designed with a user-friendly approach and it can be accessed easily. The adaptation of these resources will aid the government in reducing their burden of the healthcare units by engaging patients in beneficial services by using mobile-based healthcare services. The promotion and training of the significance and use of these services can assist the individuals to make most of these applications by staying up at home and connecting to the doctor without making too much effort. The emergence of $\mathrm{m}$ health services has triggered the feasibility of people by attaining internet-based clinical practitioner services.

This study provides assistance on being aware of current knowledge, opportunities, strengths and shortcomings about the use of e-Health and m-Health.

\section{Recommendations}

There is currently a great deal of improvement and advancement required for the digitisation of health service and m-health. The expectations of the individual and the patients are yet to be fulfilled in providing health services. Lack of physical interactions with the doctors is still an issue in m-health service. Security and trust are amongst factors that may affect the accountability for determining 
the intention of the individuals to use $\mathrm{m}$ health services. The opportunities to attract the individuals', as the current generation being tech savvy can enhance the innovation of m-health services such as the "visits, digitisation of electronic medical records (EMR), data analytics, and mobile applications for patient engagement".

Therefore, it is recommended for health authorities and policy makers to bring more advancement in the digital healthcare service, and this can be achieved through:

- Finding technological solutions to ensure that it will be real-time responses in order to solve issues and complaints of patients.

- Employing artificial intelligence that can achieve a revolution in mobile application tools for detecting the symptoms with great accuracy and also suggesting medications.

- Finding solutions that can increase healthcare data security.

\section{Bibliography}

- $\quad$ Al Ketbi. L.M.B., E Al Deen. S.Z. (2018). The attitudes and beliefs of general practitioner towards clinical practice guidelines: a qualitative study in Al Ain, United Arab Emirates. Asia Pacific family medicine, 17(1). Retrieved August 31, 2019 From: https://www.ncbi.nlm.nih.gov/pmc/arti cles/PMC5975561/pdf/12930_2018_Articl e_41.pdf

- $\quad$ Alkraiji, A., Osama, E. H., \& Fawzi, A. M. (2014). Health informatics opportunities and challenges: Preliminary study in the cooperation council for the Arab States of the Gulf. Journal of Health Informatics in Developing Countries, 8(1), 23-27.

- Alloghani. M., Hussain, A., Al-Jumeily, D. and Abuelma'atti, O. (2015). December. Technology Acceptance Model for the Use of M-Health Services amongst health-related users in UAE. In 2015 International Conference on Developments of E-Systems Engineering (DeSE) (pp. 213-217). IEEE.

- Aungst. TD., Clauson. KA., Misra. S., Lewis. TL., \& Husain. I. (2014). How to identify, assess and utilize mobile medical applications in clinical practice. Int J Clin Pract 2014; 68(2):155-62.
- Benharref. A., \& Serhani. M.A. (2013). Novel cloud and SOA-based framework for E-Health monitoring using wireless biosensors. IEEE Journal of biomedical and health informatics, 18(1), pp.46-55.

- Bhatt. C.M. E Peddoju. S.K. (eds). (2016). Cloud Computing Systems and Applications in Healthcare. IGI Global.

- Bodolica. V., E Spraggon. M. (2019). Toward patient-centred care and inclusive health-care governance: a review of patient empowerment in the UAE. Public Health, Vol 169, pp.114124.

- Clarke. K. (2015, March 18). UAE going digital for better healthcare. Retrieved March 24, 2019, From: https://www.khaleejtimes.com/nation/u ae-health/uae-going-digital-for-betterhealthcare

- DHA (Dubai Health Authority. (2019). Retrieved on 22 August, 2019 From: https://www.dha.gov.ae/en/pages/view allsmartapplications.aspx

- Export.gov. (2019). United Arab Emirates Healthcare Services. Retrieved August 31, 2019 From: https://www.export.gov/article?id=Unit ed-Arab-Emirates-Healthcare-Services

- Eysenbach. G. (2001, April-June). What is e-health? Journal of Medical Internet Research, 3(2), Retrieved March 23, 2019, From:

https://www.ncbi.nlm.nih.gov/pmc/arti cles/PMC1761894/

- Gallos. P., E Mantas. J. (2017, June). Travelers' Perceptions about m-Health Technology. In 2017 IEEE 30th International Symposium on Computer-Based Medical Systems (CBMS) (pp. 177-178). IEEE.

- Mohap (Ministry of Health \& Prevention (2019) Retrieved on August 31, 2019 From: https://www.mohap.gov.ae/en/services /Pages/e-Services.aspx?IsEService

- Harous. S., El Menshawy. M., Serhani. M.A., $\mathcal{E}$ Benharref. A. (2018). Mobile health architecture for obesity management using sensory and social data. Informatics in Medicine Unlocked, Vol 10, pp.27-44.

- Henderson. J. (2017, Sep 29). MyMedicNow launches mobile healthcare app to bridge gap between patients and 
doctors. Global Healthcare Blog. Retrieved August 20, 2019: https://www.healthcareglobal.com/techn ology/mymedicnow-launches-mobilehealthcare-app-bridge-gap-betweenpatients-and-doctors

- IMS Institute for Healthcare Informatics. (2013). Patient Apps for Improved Healthcare from Novelty to Mainstream. Retrieved August 28, 2019, From: http://moodle.univ-

lille2.fr/pluginfile.php/215345/mod_reso urce/content/0/IIHI_Patient_Apps_Repo rt.pdf.

- Jihad. A.J., Mathew. S.S., Paul. S. E Pushpalatha. D.P. (2016, November). Continuous health monitoring using smartphones - A case-study for monitoring diabetic patients in UAE. In 2016 12th International Conference on Innovations in Information Technology (IIT) (pp. 1-5). IEEE.

- Mack. H. (2017, January 06). 31 new digital health tools showcased at CES 2017. Retrieved March 24, 2019, From: https://www.mobihealthnews.com/conte nt/31-new-digital-health-tools-

showcased-ces-2017

- Moumtzoglou. A. (2014). Cloud computing applications for quality health care delivery. IGI Global.

- New UAE app helps find doctor based on medical conditions. (2019). [Blog] Retrieved from: https://www.khaleejtimes.com/news/ua e-health/new-uae-app-helps-find-doctorbased-on-medical-conditions.

- Omer. F., Hassan. N., Hussain. H., Mana. S., $\mathcal{E}$ Awad. O. (2015). Travel health, gaps in knowledge, attitudes, and practices amongst Dubai travellers, Dubai UAE. Int J Prev Med Res, 1(3), pp.126-131.

- Paho e-Health. (2019, March 12). eHealth Components. Retrieved March 24, 2019, from

https://www.paho.org/ict4health/index. php?option=com_content\&view $=$ article\&i $\mathrm{d}=80$ :components\&Itemid=0\&lang=en.

- research2guidance. (2019). The mHealth apps market is getting crowded. research2guidance. [online] Retrieved from:

https://research2guidance.com/mhealthapp-market-getting-crowded-259000mhealth-apps-now/

- Samihah Zaman. S. (2019). Mobile health apps will reduce burden on hospitals: expert. [Gulf News Online]. Retrieved from:

https://gulfnews.com/uae/health/mobil e-health-apps-will-reduce-burden-onhospitals-expert-1.1343822

- Samsung. (2019). Samsung Health. Retrieved 2019, from https://www.samsung.com/global/galax y/apps/samsung-health/

- Tradearabia.com. (2019). New mobile healthcare app launched in UAE. [online] Retrieved from: http://www.tradearabia.com/news/HE AL_330936.html

- Venot. A., Burgun. A., \& Quantin. C. (2016). Medical Informatics, e-Health: Fundamentals and Applications. Springer Editions.

- Zubair. M.F., Jahan. H., Rahaman. S., E Raina. R. (2016). M-Health an Emerging Trend An Empirical Study. Computer Science and Information Technology (CS \& IT), AIRCC, pp.167-174.

- Zubaydi. F., Saleh. A., Aloul. F., $\mathcal{E}$ Sagahyroon. A. (2015, November). Security of mobile health (mHealth) systems. In 2015 IEEE 15th International Conference on Bioinformatics and Bioengineering (BIBE) ( $p p$. 1-5). IEEE. 